\title{
Prevalência e controle de hanseníase: pesquisa em uma ocupação urbana de São Luís, Maranhão, Brasil
}

Prevalence and control of Hansen's disease: a research in an urban occupation area of São Luis, Maranhão state, Brazil

Prevalencia y control de la lepra: investigación en una ocupación urbana de São Luís, Maranhão, Brasil

Marcos Antonio Barbosa Pacheco. Centro Universitário do Maranhão (CEUMA). São Luís, MA, Brasil. mmmarco@terra.com.br (Autor correspondente) Monica Lice Leite Aires. Centro Universitário do Maranhão (CEUMA). São Luís, MA, Brasil. monica.leite@hotmail.com

Emanuelle Samary Seixas. Centro Universitário do Maranhão (CEUMA). São Luís, MA, Brasil. emanuelle.samary@hotmail.com

\section{Resumo}

Objetivo: Investigar a prevalência de incapacidades em pacientes com Hanseníase em três Unidades Básicas de Saúde (UBS) de São Luís no Maranhão e discutir preliminarmente possíveis modos de controle da doença. Métodos: Foi realizado estudo retrospectivo descritivo por meio dos prontuários de todos os pacientes hansenianos ( $n=57$ ) cadastrados nas UBS, no período correspondente aos anos de 2008 a 2009. As variáveis coletadas foram: idade, sexo, aspectos e situação clínica dos pacientes (GIF- Grau de incapacidade física, classificação operacional e forma clínica). Resultados: Foram encontrados 57 prontuários de pacientes hansenianos, dos quais 24 continham alguma alteração quanto ao grau de incapacidade dos pacientes. Entre aqueles que tinham algum grau de incapacidade física, 8 (33,3\%) encontravam-se entre 21 e 30 anos, 2 (8,3\%) tinham menos de 10 anos, 1 paciente (4,1\%) tinha mais de 60 anos. Com relação ao sexo, 15 eram homens (62,5\%) e 9 eram mulheres (37,5\%). Quanto à classificação operacional da doença, três casos eram paucibacilares e 21 multibacilares. Dos 57 casos estudados, 33 usuários apresentaram GIF 0; 17 pacientes, GIF I; e 7 pacientes, GIF II. A prevalência de incapacidades físicas em pacientes com hanseníase neste estudo foi de 42,1\%, correspondendo a 24 pacientes. Conclusão: 0 percentual de 12,28\% para GIF II sugere que são necessárias medidas de tratamento e reabilitação com uma abordagem mais específica e foco nos serviços de atenção primária, para se evitar o agravamento da enfermidade e permitir o controle das incapacidades.

\section{Abstract}

Objective: To study the prevalence of patients with disability owing to leprosy in three Primary Healthcare Centres (PHC) in São Luis, Maranhão state and to discuss possible ways of disease control. Methods: A retrospective descriptive study based on medical records of all leprosy patients $(\mathrm{n}=57)$ registered in these PHCs between 2008 and 2009. The following variables were analyzed: age, sex, aspect and clinical status (DPD - Degree of Physical Disability, operational classification and clinical form). Results: Fifty-seven medical records of patients with leprosy were found, and 24 of them contained some alteration in the DPD of patients. Among those who presented some degree of disability, eight patients (33.3\%) were between 21 and 30 years old, 2 (8.3\%) were under than 10 years of age, and 1 patient (4.1\%) was over 60 years old. Regarding sex, 15 were men (62.5\%) and 9 were women (37.5\%). With respect to the operational classification of disease, 3 cases were paucibacillary and 21 were multibacillary. Of the 57 cases, 33 patients presented DPD 0; 17 patients showed DPD I; and 7 patients presented DPD II. The prevalence of physical disabilities in leprosy patients in this study was $42.1 \%$, corresponding to 24 patients. Conclusion: The prevalence of $12.28 \%$ of DPD II patients suggests that treatment and rehabilitation measures are needed, with a more specific approach and focus on primary care services to prevent the worsening of this disease and allow the control of its disabilities.
Palavras-chave:

Prevalência

Hanseníase

Medicina de Família e Comunidade Atenção Primária à Saúde

Keywords:

Prevalence

Leprosy

Family Practice

Primary Health Care
Fonte de financiamento: declaram não haver.

Parecer CEP:

Universidade CEUMA n ${ }^{\circ}$ 155.121.

Conflito de interesses: declaram não haver. Recebido em: 18/01/2013 Aprovado em: 14/09/2013 
Resumen

Objetivo: Investigar la prevalencia de las discapacidades en los pacientes con lepra en tres Unidades Básicas de Salud (UBS) de São Luís de Maranhão, y preliminarmente discutir posibles formas de controlar la enfermedad. Métodos: Se realizó un estudio retrospectivo descriptivo a través de los historiales clínicos de todos los pacientes con lepra ( $n=57$ ) registrados en las UBS, en el período correspondiente a los años que van de 2008 a 2009. Las variables recogidas fueron: edad, sexo, aspectos y estado clínico de los pacientes (GDF - Grado de discapacidad física, clasificación operativa y forma clínica). Resultados: Se encontraron 57 registros médicos de pacientes con lepra, de los cuales 24 contenían alguna alteración relacionada con el grado de discapacidad de los pacientes. Entre los que tenían algún grado de discapacidad física, 8 (33,3\%) tenían entre 21 y 30 años, 2 (8,3\%) tenían menos de 10 años, y 1 paciente (4,1\%) tenía más de 60 años. Con respecto al sexo, 15 eran hombres (62,5\%) y 9 eran mujeres (37,5\%). En cuanto a la clasificación operativa de la enfermedad, tres casos fueron paucibacilares y 21 multibacilares. De los 57 casos estudiados, 33 usuarios presentaron GIF 0; 17 pacientes GIF I; y 7 pacientes GIF II. La prevalencia de las discapacidades físicas en pacientes con lepra en este estudio fue del 42,1\%, que corresponde a 24 pacientes. Conclusión: El porcentaje de 12,28\% para GIF II sugiere que se necesitan medidas de tratamiento y rehabilitación con un enfoque más específico y focalizado en los servicios de atención primaria, para impedir el empeoramiento de la enfermedad y permitir el control de las discapacidades.

\author{
Palabras clave: \\ Prevalencia \\ Lepra \\ Medicina Familiar y Comunitaria \\ Atención Primaria de Salud
}

\section{Introdução}

A hanseníase é uma doença infectocontagiosa, de evolução lenta, potencialmente incapacitante, que se manifesta principalmente por meio de sinais e sintomas dermatoneurológicos: lesôes na pele e nos nervos periféricos, com alteraçôes significativas de sensibilidade e surgimento de deformidades. O agente causal da doença é o Mycobacterium leprae que é eliminado pelas vias aéreas superiores. O período de incubação é variável, de 2 a 7 anos, em alguns casos com períodos de incubação de 7 meses e de mais de 10 anos. Seu reservatório é o homem e a transmissão é predominantemente domiciliar. ${ }^{1}$

O Brasil está entre os países mais endêmicos para a doença no mundo, com 33.955 novos casos em 2011 e apesar da redução da prevalência no decorrer dos anos, a hanseníase continua sendo um problema de saúde pública no país. A prevalência da doença é de 1,54/10000 habitantes, ainda inferior à meta de menos de 1 caso por 10.000 habitantes, instituída pela Organização Mundial da Saúde (OMS). ${ }^{2}$

Cerca de 20\% de todos os novos casos apresentam algum grau de incapacidade física no momento do diagnóstico, e outros $15 \%$ irão desenvolvê-las mesmo que todas as ações de saúde sejam realizadas adequadamente, seja no tratamento das neurites, dos estados reacionais e mesmo com a poliquimioterapia. ${ }^{3}$ Em 2010, o coeficiente de detecção de casos com grau 2 de incapacidade foi de 1,2/100 mil habitantes para as duas áreas mais endêmicas (regióes Norte e Centro-Oeste) e esse coeficiente mostrou-se o dobro da média nacional. ${ }^{4}$

Atualmente, a prevenção das deficiências e das incapacidades físicas resultantes da doença é realizada por meio do diagnóstico e do tratamento precoce. O tratamento da hanseníase compreende: quimioterapia específica; supressão dos surtos reacionais, com corticoides e talidomida; prevenção de incapacidades físicas; reabilitação física e psicossocial. A poliquimioterapia (PQT) com rifampicina, dapsona e clofazimina é a base do tratamento. 5

Contudo, apesar de o esquema terapêutico possibilitar a cura, podem ocorrer reaçóes durante o tratamento e após a alta, assim como quando a doença não é diagnosticada e tratada precocemente, o que possibilita quadros de neurite e, caso os pacientes não recebam orientaçóes e tratamento específicos, pode gerar incapacidades. ${ }^{5}$

Essas reaçóes sáo divididas em dois tipos: a) Tipo1 - ocorrem em pacientes com algum grau de imunidade celular, como os tuberculoides e dimorfos; e b) Tipo 2 - reaçóes mediadas por anticorpos, que ocorrem nos Virchowianos e também em alguns dimorfos. A manifestação clínica mais frequente da reação tipo 2 é o eritema nodoso hansênico. ${ }^{6}$ As reaçôes do tipo 1, também denominadas reaçôes reversas, são causa frequente de incapacidades, o que torna de suma importância as avaliaçóes periódicas dos pacientes em tratamento, mesmo na ausência de qualquer queixa. ${ }^{7}$

A OMS, com o objetivo de sistematizar o estudo das incapacidades geradas pela hanseníase, criou um formulário para anotaçôes e detalhamento dos diferentes graus em cada paciente, considerando inicialmente três graus de incapacidade, em ordem crescente, segundo o acometimento de mãos, pés e olhos: o grau zero corresponde à ausência de incapacidades devido à hanseníase, e os graus 1 e 2 decorrem de alteraçóes sensitivas e/ou motoras de gravidade crescente. ${ }^{8}$

No Brasil, os estudos a respeito das incapacidades em pacientes com hanseníase na população geral ainda são escassos. Diante dessa carência de dados, torna-se relevante levantar a prevalência de incapacidades nos pacientes com Hanseníase, determinar a tipologia e graus de incapacidade desses pacientes e verificar a distribuição quanto ao gênero, faixa etária e modo de entrada. 
A ideia de se trabalhar mais especificamente com a prevalência de incapacidade provocada pela doença é porque esta se constitui em bom indicador para apontar diagnósticos tardios. Contudo, além de uma descrição da prevalência de incapacidades e suas correlaçôes, pontua-se, neste artigo, o papel da atenção primária no âmbito do controle de endemias, tomando como referência empírica, o caso da hanseníase no contexto de investigação e atuação dos autores deste estudo.

Quanto ao perfil local da hanseníase, dados da Secretaria Municipal de Saúde (SEMUS) dáo conta de que no ano de 2011 a prevalência de hanseníase no município de São Luís foi de 6,5 casos para cada grupo de dez mil pessoas, o que coloca o município na condição de hiperendêmico, juntamente com outros municípios do Estado do Maranhão, unidade da federação também considerada hiperendêmica. ${ }^{2}$

No caso específico desta investigação sobre incapacidade provocada por hanseníase, este estudo teve como objetivo estudar a prevalência de incapacidades em usuários das três UBS da Comunidade da Cidade Olímpica, na cidade de São Luís, no Estado do Maranhão. A presente pesquisa tratou, portanto, de mapear a prevalência de hanseníase em um determinado território assistencial e, secundariamente, suscitar alternativas para seu enfrentamento.

\section{Métodos}

O presente artigo é decorrente de um trabalho de pesquisa desenvolvido em três Unidades Básicas de Saúde (UBS) localizadas em uma ocupação urbana no município de São Luís (MA), cujo objetivo foi estudar a prevalência de incapacidades em pacientes com hanseníase inscritos nessas Unidades. A comunidade chama-se Cidade Olímpica, em alusão às olimpíadas de 1996, ano em que se deu a ocupação (desordenada) do bairro que atualmente conta com uma população estimada em mais de 72 mil habitantes, cujas famílias são originárias das mais diversas regiôes do Estado. ${ }^{9}$

Esta comunidade possuía (no período relativo aos questionários pesquisados) e ainda possui uma cobertura assistencial da Estratégia Saúde da Família (ESF) em torno de 45\%. Tomando-se como parâmetro a relaçáo média de 3.500 pessoas por equipe, e considerando-se a presença de apenas nove equipes (três em cada UBS) para uma população estimada de 72 mil habitantes, que deveria ter no mínimo vinte equipes, as nove equipes representam, neste ponto de corte, uma cobertura de menos da metade do que seria minimamente desejável. ${ }^{10}$

Realizou-se um estudo retrospectivo descritivo que se utilizou de pesquisa documental em prontuários de todos os pacientes hansenianos $(\mathrm{n}=57)$ cadastrados nas três UBS no distrito sanitário do Tirirical, São Luís do Maranhão, no período correspondente aos anos de 2008 a 2009. Os critérios para inclusão foram pacientes em tratamento nos anos de 2008 e 2009, ou seja, todos os pacientes diagnosticados e/ou assistidos nesse período, cadastrados no programa de controle da hanseníase das Unidades de Saúde citadas. Contudo, a pesquisa concentrou-se nos usuários que apresentaram algum grau de incapacidade.

Extraíram-se, dos registros nos prontuários, variáveis relacionadas aos dados pessoais dos pacientes (idade e sexo) e aos aspectos e situação clínica, tais como: modo de entrada, classificação operacional (paucibacilar ou multibacilar) e Grau de Incapacidade Física (GIF 0, 1 ou 2) no momento do diagnóstico. Os achados foram transcritos para uma Planilha Excel ${ }^{\circledR}$ e todos os casos de incapacidade física foram categorizados conforme critérios estabelecidos na Portaria do Ministério da Saúde (MS) n. ${ }^{\circ}$ 3.125, de 7 de outubro de 2010 que dispóe sobre as Diretrizes para Vigilância, Atenção e Controle da hanseníase, ${ }^{11}$ conforme quadro a seguir (Quadro 1 ).

Quadro 1.Caracterização de incapacidades de acordo com critérios estabelecidos pela portaria do Ministério da Saúde 3.125/2010.

\begin{tabular}{cl}
\hline Grau de incapacidade & \multicolumn{1}{c}{ Características } \\
\hline 0 & Nenhum problema com olhos, mãos ou pés devido à hanseníase. \\
1 & Diminuição ou perda da sensibilidade nos olhos. Diminuição ou perda da sensibilidade protetora nas mãos e /ou nos pés. \\
2 & Olhos: lagoftalmo e/ou ectrópio; triquíase; opacidade corneana central; acuidade visual menor que 0,1 ou incapacidade de contar dedos a \\
& 6m de distância. Mãos: lesões tróficas e/ou lesões traumáticas; garras; reabsorção; mão caída. Pés: lesões tróficas e/ou traumáticas; garras; \\
& reabsorção; pé caído; contratura do tornozelo.
\end{tabular}

Fonte: Portaria MS 3.125/2010.11 
Foi feita a análise descritiva dos dados, com indicação de frequência e cálculo percentual. Os dados foram obtidos a partir dos prontuários, preservando-se a identidade dos pacientes, de acordo com as exigências da Resolução 196/96 do Conselho Nacional de Saúde. O presente trabalho foi encaminhado ao Comitê de Ética e Pesquisa com seres humanos da Universidade Centro Universitário do Maranhão (CEUMA) e foi aprovado sob parecer favorável de no 155.121 , com dispensa do Termo de Consentimento Livre e Esclarecido (TCLE), por se tratar de estudo utilizando dados de prontuários.

\section{Resultados}

Do conjunto de um total de 57 prontuários de pacientes hansenianos analisados, 24 (42,1\%) eram portadores de algum grau de incapacidade física. Entre aqueles que tinham algum grau de incapacidade física, $8(33,3 \%)$ encontravam-se entre 21 e 30 anos; 2 (8,3\%) tinham menos de 10 anos; 1 paciente $(4,1 \%)$ tinha mais de 60 anos. Em relação ao sexo, 15 pacientes eram homens $(62,5 \%)$, enquanto 9 eram mulheres $(37,5 \%)$ (Tabela 1$)$.

Tabela 1. Distribuição quanto ao sexo e grau de incapacidades em pacientes com hanseníase registrados em 3 UBS de São Luís, no período de 2008 a 2009.

\begin{tabular}{|c|c|c|c|c|c|c|}
\hline \multirow[t]{3}{*}{ Grau de incapacidade } & \multicolumn{4}{|c|}{ Sexo } & \multirow{2}{*}{\multicolumn{2}{|c|}{ Total }} \\
\hline & \multicolumn{2}{|c|}{ Feminino } & \multicolumn{2}{|c|}{ Masculino } & & \\
\hline & $\mathrm{N}$ & $\mathrm{n} \%$ & $\mathrm{~N}$ & $\mathrm{n} \%$ & $\mathrm{~N}$ & $\mathrm{n} \%$ \\
\hline 0 & 20 & 35,1 & 13 & 22,8 & 33 & 57,9 \\
\hline 1 & 06 & 10,5 & 11 & 19,3 & 17 & 29,8 \\
\hline 2 & 03 & 05,2 & 04 & 07,1 & 07 & 12,3 \\
\hline Total & 29 & 50,8 & 28 & 49,2 & 57 & 100,0 \\
\hline
\end{tabular}

Quanto ao modo de entrada, isto é, a condição ou modo de inscrição do usuário, a maior parte do grupo com incapacidades correspondeu a "casos novos" (58,33\%); 1 caso correspondendo a 4,16\% dos pacientes apresentou-se como "recidiva", o qual ocorreu com um caso "encaminhado"; um caso "transferido" e outro como "contactante". Em 6 prontuários (25\%) a informação sobre o modo de entrada estava ausente (Tabela 2).

Tabela 2. Distribuição da frequência e percentual, segundo o modo de entrada, dos pacientes com incapacidades em hanseníase registrados em 3 UBS de São Luís, no período de 2008 a 2009.

\begin{tabular}{lcc}
\hline \multicolumn{1}{c}{ Modo de entrada } & $\mathrm{N}$ & $\mathrm{n} \%$ \\
\hline Casos novos & 14 & 58,33 \\
Não informado & 6 & 25 \\
Encaminhado & 1 & 4,16 \\
Transferência & 1 & 4,16 \\
Contactante & 1 & 4,16 \\
Recidiva & 1 & 4,16 \\
\hline
\end{tabular}

Quando analisada a classificação operacional da doença, foram encontrados 3 casos paucibacilares (12,5\%): 1 indeterminado (4,16\%) e 2 tuberculoides (8,33\%), e 21 multibacilares (87,5\%): sendo 18 dimorfa (75\%) e 3 virchowiana (12,5\%) (Tabela 3).

Tabela 3. Distribuição de número e percentual por classificação operacional, dos pacientes com incapacidades em hanseníase registrados em 3 UBS de São Luís, no período de 2008 a 2009.

\begin{tabular}{lcc}
\hline Classificação Operacional & $\mathrm{N}$ & $\mathrm{n} \%$ \\
\hline Paucibacilar & 3 & 12,5 \\
Indeterminado & 1 & 4,16 \\
Tuberculoide & 2 & 8,33 \\
Multibacilar & 21 & 87,5 \\
Dimorfa & 18 & 75 \\
Virchowiana & 3 & 12,5 \\
\hline
\end{tabular}




\section{Discussão}

Nas três UBS pesquisadas, o número total de hansenianos inscritos para tratamento no período era de 57, dos quais, como já apontado, 24 eram portadores de incapacidades físicas. O número de inscritos chama a atenção para a condição de hiperendemicidade, uma vez que o MS e a OMS têm por meta uma taxa de prevalência menor que 1 caso para cada grupo de 10 mil habitantes. Considerando-se que na época referida nos prontuários (2008 a 2009) a Cidade Olímpica possuía pouco mais de 60 mil habitantes, a prevalência tecnicamente aceitável seria de apenas entre seis e sete pacientes hansenianos na localidade.

Percebe-se que existe uma parcela significativa de pacientes com incapacidades, representando 42,1\% da amostra de 57 pacientes. As deformidades e incapacidades físicas são o principal problema da hanseníase, sendo o percentual de pacientes com incapacidades, um indicador do impacto sociossanitário importante da doença ${ }^{8}$ e, certamente, a baixa cobertura da ESF constitui-se em fator causal significativo.

A prevalência de incapacidades foi investigada em 2005 em 11 municípios do Paraná e atribuiu-se a alta prevalência encontrada $(49,8 \%)$, inclusive mais alta que a da presente pesquisa $(42,1 \%)$, à falta de qualificação dos profissionais e ao diagnóstico tardio dos casos. A realização de diagnóstico tardio, já com incapacidades, sugere que os serviços de saúde não estariam sendo capazes de detectar todos os doentes existentes, o que contribuiria para a permanência de casos não diagnosticados (prevalência oculta) evoluindo para deformidades, incapacidades e a continuidade da cadeia de transmissão. ${ }^{12}$

Quanto à faixa etária, as faixas que variavam entre 21 a 30 anos, 31 a 40 e 41 a 50 anos foram responsáveis respectivamente por $33,3 \%, 16,6 \%$ e 16,6\%, totalizando dois terços dos acometidos, exatamente nas faixas de maior produtividade econômica desses pacientes. Em estudo de Gomes et al. ${ }^{13}$ observa-se que é também um indicador importante na prevalência das incapacidades, o fato de haver maior número de casos na faixa economicamente ativa, e, portanto, sob risco de exclusão da cadeia produtiva de adultos e, algumas vezes, de adultos jovens. Neste trabalho, a maioria dos usuários com incapacidades foi do sexo masculino $62,5 \%$. Isso confirma o fato de que mulheres, de maneira geral, procuram mais os serviços de saúde e, neste caso, tratam mais "precocemente" sua hanseníase, evitando incapacidades. No caso específico da hanseníase, como apresenta deformidades evidentes, seria mais um fator de busca do tratamento mais cedo. As mulheres, de modo geral, se mostram mais preocupadas com a autoimagem do que os homens.

Quanto ao modo de entrada, grande parte dos hansenianos com incapacidades correspondeu a casos novos $(58,33 \%)$. No Brasil, as taxas de detecçáo de novos casos de hanseníase sofreu declínio, mas ainda permanecem altas nas regiôes Norte, Centro-Oeste e Nordeste, quando comparadas às regióes Sul e Sudeste, segundo dados e indicadores da Secretaria de Vigilância em Saúde. ${ }^{2}$

A investigação por meio de estudo bibliográfico sobre a tendência das taxas de detecção de novos casos de hanseníase em áreas endêmicas do Pacífico, Ásia, África e América Latina, apontou que a única das áreas estudadas em que houve consistente aumento da detecção da doença nos últimos 20 anos foi a América Latina. ${ }^{14}$ As tendências de aumento da detecção em São Luís e no Brasil corroboram com esses resultados.

Neste estudo, quanto ao grau de incapacidades, 33 pacientes $(57,89 \%)$ apresentaram Grau de Incapacidade Física 0 (zero); 17 pacientes $(29,82 \%)$ apresentaram alteração de sensibilidade protetora - GIF 1; e 7 pacientes (12,28\%) possuíam deformidades instaladas - GIF 2, ou seja, dos referidos casos 24 pacientes $(42,1 \%)$ apresentaram alguma alteração quanto ao grau de incapacidade.

De acordo com diferentes estudos realizados, é de fácil percepção que o GIF é variável. Em uma investigação realizada na faculdade de medicina de Ribeirão Preto (SP), com o intuito de prevenir a incapacidade na hanseníase, dos 18 pacientes selecionados, 6 apresentaram GIF 0, 14 GIF 1, e 6 pacientes GIF $2 .{ }^{15} \mathrm{Em}$ uma pesquisa realizada em um centro de referência de Campo Grande, avaliaram-se 282 prontuários de hansenianos, no período de 2000 a 2002, e os autores concluíram que $31,2 \%$ foram caracterizados no GIF 1 e 16,1\% no GIF 2, totalizando 47,3\% dos casos que possuíam algum grau de incapacidade com alteração de sensibilidade e/ou física. ${ }^{16}$

No estudo de Gomes et al. ${ }^{13}$ realizado em um centro de referência, no período de novembro de 2003 a janeiro de 2005, com 61 pacientes, encontraram-se 59\% com GIF 0; 23\% GIF 1; e 18\% com GIF 2. Em estudo retrospectivo realizado no sul do País, dos 92,6\% pacientes avaliados, foram encontrados 57,4\% de pacientes com GIF 0; 27,8\% GIF 1; e 14,8\% com GIF $2 .{ }^{17}$ 
Por meio destes estudos, verifica-se que a alta prevalência de indivíduos com incapacidades físicas está presente em diversas regiôes, de norte a sul do País. Sendo assim, torna-se de extrema importância identificar e investir na prevenção das incapacidades. Uma conduta que pode prevenir e até reverter sequelas físicas é a avaliação de incapacidades no início do tratamento, pois se o usuário apresentar nervos acometidos, os riscos de desenvolver incapacidades sáo maiores. Portanto, os programas de controle da hanseníase devem ser criteriosos na avaliação inicial. ${ }^{14}$

Neste estudo, verificou-se que o percentual de casos com deformidades físicas grau 2 (12,28\%), conforme Tabela 3, está acima do preconizado pelo Ministério da Saúde (menor que 5\%). A razão do percentual não desejado evidencia a presença de diagnósticos tardios e falta de ações para prevenção de incapacidades dela decorrentes. Nessas situaçóes de grande proporção de GIF 2, medidas de tratamento e reabilitação devem ter uma abordagem mais específica, com o intuito de evitar o agravamento da enfermidade e permitir o controle das incapacidades.

Atividades voltadas para a prevenção são essenciais, considerando-se o impacto que as incapacidades causam na qualidade de vida de seus portadores. Segundo estudo realizado no ambulatório de Hansenologia do Hospital Universitário Clementino Fraga Filho da Universidade Federal do Rio de Janeiro (HUCFF-UFRJ), mais de 50\% dos pacientes acometidos com GIF 1 ou 2 apresentaram escore grave ou muito grave, no Dermatology Life Quality Index (DLQI), contrastando com os pacientes que não possuem incapacidade física, o que evidencia o comprometimento da qualidade de vida de pacientes com GIF. ${ }^{18}$

A hanseníase causa grande prejuízo para as atividades da vida diária e às relaçóes interpessoais, provocando sofrimento que ultrapassa a dor e o mal-estar estritamente vinculados ao prejuízo físico, com grande impacto social e psicológico. O controle de endemias como hanseníase e tuberculose pode ser muito efetivo a partir da consolidação e fortalecimento da Atençáo Primária à Saúde (APS). De fato, o sucesso da estratégia de controle da hanseníase e de outras doenças transmissíveis e mesmo não transmissíveis, consiste em equipar e garantir infraestrutura aos serviços de saúde e, mais ainda, preparar os profissionais para transformar o cenário epidemiológico e o quadro sociossanitário dessas doenças no seu território de atuação, proporcionando-lhes capacitação, utilizando técnicas de interação teoria-prática, ensino, serviço e comunidade como instrumentos metodológicos eficientes, na mesma linha que vem apontando a nova Política Nacional de Atenção Básica (PNAB), da necessidade da máxima integração de diferentes competências no nível primário da assistência:

[...] a presença de diferentes formaçôes profissionais assim como um alto grau de articulação entre os profissionais é essencial, de forma que não só as açôes sejam compartilhadas, mas também tenha lugar um processo interdisciplinar no qual progressivamente os núcleos de competência profissionais específicos vão enriquecendo o campo comum, ampliando assim a capacidade de cuidado. ${ }^{19}$

A partir de uma análise dos dispositivos da Portaria 3.125 de 7 de outubro de $2010,{ }^{11}$ que aprova as diretrizes para vigilância, atenção e controle da hanseníase no Brasil, é possível extrair alguns elementos que reforçam a concepção de que doenças como estas podem (e devem) ser controladas perfeitamente no âmbito da atenção primária, em espaços ambulatoriais e inseridos no contexto comunitário. O conteúdo normativo da referida Portaria é resultado de uma experiência acumulada no Brasil no cuidado e assistência a esse agravo.

Entre os elementos que reforçam a "competência" de uma assistência comunitária no controle da hanseníase podem ser elencados:

- o diagnóstico é essencialmente clínico e epidemiológico;

- a baciloscopia tem por finalidade classificar o caso, é um exame relativamente simples e sua negatividade não exclui o diagnóstico;

- o tratamento está bem definido na portaria citada, sendo duas modalidades operacionais: paucibacilar e multibacilar;

- mesmo as complicaçóes da doença - preponderantemente "reaçôes hansênicas" - quando ocorrem na sua grande maioria podem ser conduzidas no âmbito da atenção primária, principalmente com corticoides por pouco tempo e "desmame" regressivo.

Portanto, é importante ressaltar essa "competência" da atenção primária como uma possibilidade bem exequível do ponto de vista técnico operacional e com impactos altamente positivos. Isso porque ainda existe muita resistência de alguns profissionais médicos e enfermeiros no manejo clínico da hanseníase, bem como da tuberculose, no atendimento primário. Isso ficou evidente nas "rodas de conversa" informais realizadas pelos pesquisadores com profissionais das equipes de saúde das unidades pesquisadas, cuja tendência é o "encaminhamento" desses pacientes para "especialistas". 
Neste caso específico, a pesquisa motivou a iniciativa de instalação de um projeto de intervenção na comunidade, a ser desenvolvido com o escopo do melhor controle do agravo, sobretudo, a partir da socialização de seus dados entre os profissionais de saúde das unidades. De fato, em algumas "rodas de conversa" informais, pesquisadores e profissionais das unidades motivaram-se para um trabalho conjunto com a Universidade originária da pesquisa, no sentido de estabelecer um projeto-piloto de apoio ao controle da hanseníase naquela comunidade, dada a gravidade da situaçáo e impacto que poderia gerar. Com isso, há um potencial para a integração das dimensôes da docência e da assistência em uma articulação mais do que necessária entre a pesquisa acadêmica e o serviço de saúde. Ademais, pode-se falar também em pesquisa propositiva, na medida em que o projeto de intervençáo constitui-se em um programa de apoio ao controle da hanseníase e da tuberculose na comunidade pesquisada, cujos indicadores para estes agravos carecem de uma melhoria significativa.

\section{Conclusão}

A situaçáo de hiperendemicidade encontrada na pesquisa, bem como a alta prevalência de incapacidade, reflete entre outros fatores, a baixa cobertura da ESF e também a necessidade de qualificação assistencial, na medida em que foi muito referido pelos próprios profissionais, não apenas o "encaminhamento" do paciente, mas, às vezes, o reconhecimento da necessidade de mais capacitaçóes para o manejo da doença de Hansen.

A presença de incapacidades indica detecção tardia da doença, tratamento inadequado e conhecimento deficiente, tanto dos profissionais de saúde quanto dos pacientes. A complexidade de enfrentamento de uma doença como a hanseníase leva a refletir e redirecionar os modos de controle para uma medicina cada vez mais comprometida com a realidade sociossanitária dos territórios onde pretende intervir. Segundo a nova PNAB: "Essa organização pressupóe o deslocamento do processo de trabalho centrado em procedimentos profissionais para um processo centrado no usuário, onde o cuidado do usuário é o imperativo ético-político que organiza a intervenção." 18

\section{Referências}

1. Rouquayrol MZ. Epidemiologia \& Saúde. Cidade: Ed. Medsi; 2010. 708 p.

2. Ministério da Saúde (BR). Indicadores epidemiológicos e operacionais de hanseníase. Brasília: Secretária de Vigilância em Saúde; 2000-2011. Dados disponíveis em 24/04/2012.

3. Ramos JM, Souto FJ. Incapacidade pós-tratamento em pacientes hansenianos em Várzea Grande, Estado de Mato Grosso. Rev Soc Bras Med Trop. 2010; 43(3): 293-297. http://dx.doi.org/10.1590/S0037-86822010000300016

4. Ignotti E, Paula RC. Situação epidemiológica da hanseníase no Brasil: análise de indicadores selecionados no período de 2001 a 2010 . Brasília: Secretaria de Vigilância em Saúde; 2010.

5. Ministério da Saúde (BR). Secretaria de Vigilância em Saúde. Guia de Vigilância em Saúde. 6. ed. Brasília: Ministério da Saúde; 2006.

6. Véras LS, Vale RG, Mello DB, Castro JA, Lima V, Silva KN, et al. Degree of disability, pain levels, muscle strength, and electromyographic function in patients with Hansen's disease with common peroneal nerve damage. Rev Soc Bras Med Trop. 2012; 45(3): 375-379. http://dx.doi.org/10.1590/ S0037-86822012000300018

7. Gonçalves SD, Sampaio RF, Antunes CM. Fatores Preditivos de Incapacidades em Pacientes com Hanseníase. Rev Saúde Pública. 2009; 43(2):267-74. http://dx.doi.org/10.1590/S0034-89102009000200007

8. Ministério da Saúde (BR). Guia de Hanseníase. Brasília; 2002. 49 p.

9. Afonso MC. Memória e história do direito que se faz na luta: o caso da ocupação e posse na Cidade Olímpica [Monografia]. São Luís: Universidade Estadual do Maranhão; 2011.

10. Secretaria Municipal de Saúde de São Luís (MA). Superintendência de Planejamento e Programação em Saúde. Relatório de Gestão, 2012. São Luís: SEMUS; 2013.

11. Brasil. Ministério da Saúde. Portaria MS n 3.125, de 7 de outubro de 2010. Dispõe sobre as Diretrizes para Vigilância, Atenção e Controle da Hanseníase. Brasília: Ministério da Saúde; 2010.

12. Silva RAS, Mathias TAF, Gomes EA, Lincoln PB. Avaliação do Grau de Incapacidade em Hanseníase: Uma estratégia para sensibilização e capacitação da equipe de enfermagem. Rev Latino-am Enfermagem. 2007; 16(6), 1125-1130. http://dx.doi.org/10.1590/S0104-11692007000600011 
13. Gomes CC, Pontes MA, Gonçalves HS, Penna GO. Perfil clínico epidemiológico dos pacientes diagnosticados com hanseníase em um centro de referência na região nordeste do Brasil. An Bras Dermatol. 2005; 80(Supl 3): S283-8. http://dx.doi.org/10.1590/S0365-05962005001000004

14. Ministério da Saúde (BR). Secretaria de Vigilância em Saúde. Portaria Conjunta n. 125, de 26 de março de 2009. Define as ações de controle da hanseníase e dá outras providencias. Diário Oficial da Republica Federativa do Brasil, Brasília, 27 Mar 2007. Secão 1. p. 73.

15. Rodini FC, Gonçalves M, Barros AR, Mazzer N, Elui VM, Fonseca MC. Prevenção de incapacidade na hanseníase com apoio em um manual de autocuidado para pacientes. Fisioter Pesq. 2010; 17(2): 157-66.

16. Corrêa CM, Ivo ML, Honer MR. Incapacidades em sujeitos com hanseníase em um centro de referência do centro- oeste brasileiro entre $2000-2002$. Hansenol Int. 2006; 31(2).

17. Melão S, Blanco LF, Mounzer N, Veronezi CC, Simões PW. Perfil epidemiológico dos pacientes com hanseníase no extremo sul de Santa Catarina, no período de 2001 a 2007. Rev Soc Bras Med Trop. 2011; 44(1): 79-84. http://dx.doi.org/10.1590/S0037-86822011000100018

18. Martins BD, Torres FN, Oliveira ML. Impacto na qualidade de vida em pacientes com hanseníase: correlação do Dermatology Life Quality Index com diversas variáveis relacionadas à doença. An Bras Dermatol. 2008; 83(1). http://dx.doi.org/10.1590/S0365-05962008000100005

19. Ministério da Saúde (BR). Portaria MS n².488, de 21 de outubro de 2011. Dispõe sobre a Política Nacional de Atenção Básica. Brasília: Ministério da Saúde; 2011. 